DOANH NGHIỆP NHỎ VÀ VÙ̉A: SỬ PHÂN NHÓM VÀ DẠNG THỨC CỦA NHÀ QUẢN LÝ CHỦ NHÂN

\title{
SMALL AND MEDIUM SIZED ENTERPRISES: CLUSTERING AND OWNER MANAGER'S TYPOLOGY
}

\section{Trương Minh Chương}

Khoa Quản Lý Công Nghiệp, Đại Học Bách Khoa, ĐHQG-HCM - Email: tmchuong@hcmut.edu.vn

\section{TÓM TÄT}

Doanh nghiệp nhỏ và vừa được thành lập và quản lý bởi nhà quản lý chủ nhân. Các đặc điểm tâm lý, nhân khẩu của nhà quản lý chủ nhân ảnh huởng đến hệ thống quản lý do ho thiết lập và kết quả hoạt động của doanh nghiệp. Nghiên cưu này nhằm tìm ra các cấu trúc nhóm của các doanh nghiệp này thông qua các đặc điểm nêu trên. Tù đó, xác định bộ các tiêu chí để nhận diện dạng thức nhà quản lý chủ nhân và dư đoán kết quả hoạt động của doanh nghiệp. Phuơng pháp phân tích nhóm đã được thực hiện và đã phân tích 240 doanh nghiệp thành 3 nhóm với sự khác biệt đáng kể về các yếu tố nhu đặc điểm tâm lý, đặc điểm nhân khẩu, quản trị chiến luợc, cấu trúc theo chức năng. Các yếu tố này đã tạo bộ tiêu chí nhận dạng các nhà quản lý chủ nhân và dụ đoán kết quả hoạt động của doanh nghiệp. Quan hệ giữa các yếu tố này trong mỗi nhóm cũng đã được nhận diện.

Tù khóa: Doanh nghiệp nhỏ và vùa, nhà quản lý chủ nhân, quản trị chiến lược, cấu trúc tổ chức

\section{ABSTRACT}

SMEs are established and managed by owner managers. Their personality traits, demographics impact the enterprise's managerial system and performance. This study aims at clustering the SMEs to find out the clustering structure via these stated above factors, then, defining a set of criteria to recognize the owner managers' typology and predicting the enterprise performance. Clustering method has been applied to analyze 240 enterprises into 3 clusters differentiating with each other basing on the personality traits, particularly, innovation, demographics, strategic management and organizational functional structure. These factors are encapsulated into a set of criteria for owner manager' typology definition and prediction of the enterprise performance. Relations among these factors in each cluster were also recognized.

Key words: Small and Medium sized Enterprise (SME), owner manager, strategic management, organizational structure. 


\section{GIỚI THIẸU}

Nghiên cứu về DNNVV, tập trung vào việc nhận dạng NQLCN-người thành lập và quản lý doanh nghiệp- và quan hệ giữa các đặc điểm tâm lý, nhân khẩu của NQLCN với các yếu tố nội bộ và kết quả hoạt động của doanh nghiệp [2;19]. Các nghiên cứu này đã tạo ra các kết quả không đồng nhất vì có nhiều đặc điểm tâm lý, nhân khẩu khác nhau với các định nghĩa khác nhau được khảo sát và các yếu tố nội bộ được khảo sát riêng rẻ nên không mô tả, giải thích được tính đa dạng của DNNVV [9]. Để khắc phục các hạn chế nêu trên, một số nghiên cứu đã được thực hiện theo phép tiếp cận phân nhóm để nhận diện một số nhóm đặc trưng của các DNNVV dựa trên sự kết hợp các đặc điểm của NQLCN, các yếu tố nội bộ, môi trường kinh doanh [8]. Từ đó, các yếu tố tạo sự phân nhóm các NQLCN được gọi là dạng thức của NQLCN được nhận diện. Tuy nhiên, kết quả của các nghiên cứu này cũng còn nhiều tranh luận [1] vì sự không đồng nhất trong việc chọn lựa các yếu tố để tạo dạng thức NQLCN [20; 23]. Trong nghiên cứu này, ngoài các yếu tố đặc điểm tâm lý, nhân khẩu của NQLCN, quản trị chiến lược (QTCL) và cấu trúc $(\mathrm{CT})$ là những yếu tố tạo sáng tạo cải tiến [22] được bổ sung vào để tạo dạng thức của NQLCN. Do đó, nghiên cứu này được thực hiện nhằm mục tiêu: (1) Nhận diện các nhóm đặc trưng của DNNVV dựa trên các đặc điểm tâm lý, nhân khẩu của NQLCN, QTCL, CT, môi trường và kết quả hoạt động của doanh nghiệp, (2) nhận diện các dạng thức của NQLCN và quan hệ giữa các dạng thức này với kết quả hoạt động của doanh nghiệp trong mỗi nhóm.

\section{CƠ SỞ LÝ THUYÊT VÀ PHƯƠNG PHÁP NGHIÊN CÚU}

\subsection{Cơ sở lý thuyết và khung phân tích}

Các đặc điểm tâm lý nhân khẩu của NQLCN và quan hệ của chúng với kết quả hoạt động của doanh nghiệp là nội dung ban đầu của các nghiên cứu về tinh thần doanh nhân (TTDN) và quản lý DNNVV. Có nhiều đặc điểm tâm lý khác nhau của NQLCN được khảo sát. Trong nghiên cứu này các đặc điểm như sự chấp nhận rủi ro, nhu cầu thành đạt, sự kiểm soát bản thân, sự tự tin, sự sáng tạo, sự tự chủ được khảo sát vì đó là các đặc điểm để phân biệt NQLCN có TTDN và NQLCN bình thường $[2 ; 18 ; 19 ; 21]$. Các đặc điểm nhân khẩu của NQLCN như tuổi, trình độ học vấn, chuyên ngành đào tạo, thâm niên công tác, kinh nghiệm nghề nghiệp, truyền thống gia đình, quan hệ xã hội - là những yếu tố tạo nền tảng cho sự nhận thức, đánh giá và quyết định khai thác cơ hội kinh doanh - cũng được bao gồm trong nghiên cứu này.

NQLCN sẽ xây dựng chiến lược [14; 22; 24]. Sự lựa chọn chiến lược phụ thuộc vào các đặc điểm tâm lý, nhân khẩu của NQLCN [12; 18]. Để thực hiện chiến lược đã được lựa chọn đó, NQLCN sẽ dựa vào các đặc điểm tâm lý, nhân khẩu của mình để thiết lập $\mathrm{CT}$, các quy trình làm việc cho doanh nghiệp[14; 19] phù hợp với môi trường kinh doanh [3; 7]. Dựa trên chiến lược, CT, NQLCN triển khai vận hành doanh nghiệp để tạo kết quả hoạt động cho doanh nghiệp. Do đó, chiến lược, $\mathrm{CT}$ của doanh nghiệp có liên hệ mật thiết với các đặc điểm tâm lý, nhân khẩu của NQLCN, môi trường kinh doanh và kết quả hoạt động của doanh nghiệp. Các quan hệ này tạo khung phân tích cho bài nghiên cứu này như được trình bày trong Hình 1 .

\section{Trang 26}




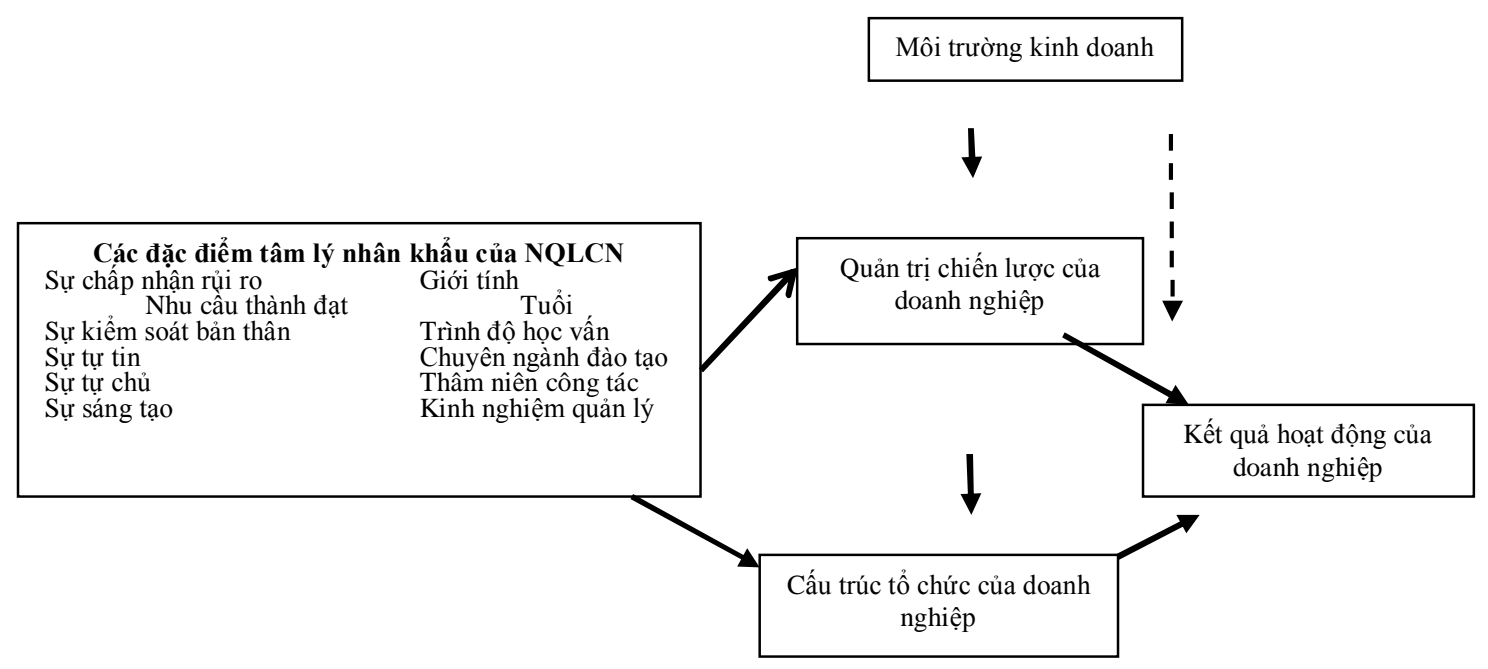

Hình 1: Khung phân tích cho bài nghiên cứu

\subsection{Phuơng pháp nghiên cúu}

Nghiên cứu được thực hiện thông qua hai bước: Kiểm định các thang đo và phân tích nhóm. Các thang đo được kiểm định thông qua phân tích nhân tố khám phá để đảm bảo tính đơn hướng, độ giá trị hội tụ và đánh giá độ tin cậy thông qua chỉ số Cronbach's Alpha [10]. Sau khi kiểm định thang đo, các giá trị của từng thang đo đối với mỗi đối tượng trong mẫu được tính toán và dùng như các biến cho việc phân tích nhóm.

Để phân nhóm, thuật toán phân nhóm hình tháp với việc phân nhóm theo nguyên tắc phát triển nhóm và phương pháp Ward sẽ được dùng [6]. Khoảng cách giữa các đối tượng trong mẫu được đo bởi bình phương khoảng cách Euclide. Để sắp xếp các đối tượng vào các nhóm tương ứng, phương pháp phân nhóm $\mathrm{K}$ means được sử dụng[6]. Sự khác biệt đáng kể giữa các nhóm được đánh giá thông qua phân tích ANOVA về sự khác biệt của trị trung bình của mỗi biến số trong mỗi nhóm [6].Thang đo sự kiểm soát bản thân, nhu cầu thành đạt, sự tự tin được xây dựng dựa trên nội hàm của các khái niệm này được định nghĩa trong nghiên cứu [18]. Các thang đo cho các khái niệm còn lại được kế thừa từ các nghiên cứu trước [5;17]. Các thang đo này đều được đo theo thang Likert 5 điểm. Tổng cộng có 43 biến số được tạo ra cho các thang đo này.

Mẫu được thu thập theo phương pháp thuận tiện. Khung lấy mẫu là các doanh nghiệp tại Thành Phố Hồ Chí Minh. Dữ liệu được thu thập bằng bảng khảo sát được gởi trực tiếp đến các doanh nghiệp. Kích thước mẫu được dự tính là trên 200. Tổng cộng có 1020 bảng khảo sát được phát ra, thu hồi về được 240 bảng hợp lệ. Tỷ lệ hồi đáp là $23,5 \%$. Dữ liệu được phân tích bằng phần mềm SPSS phiên bản 20.

\section{KẾT QUẢ VÀ THẢO LUẬN}

\subsection{Kết quả nghiên cứu}

Kiểm định thang đo: Kết quả phân tích nhân tố khám phá và độ tin cậy Cronbach's Alpha được trình bày trong Bảng 1 . Sau khi loại 12 biến, các thang đo đảm bảo tính đơn hướng, độ giá trị hội tụ, độ tin cậy. Giá trị của mỗi thang 
đo cho mỗi đối tượng trong mẫu được tính toán, được chuẩn hóa và mã hóa thành các biến số V1 đến V10 tương ứng với 10 khái niệm và được dùng trong phân tích nhóm[10].

Phân tích nhóm: Mẫu được chia làm hai phần bằng nhau một cách ngẫu nhiên. Kết quả phân tích nhóm theo phương pháp Ward cho cả hai phần được trình bày trong 7 cột bên trái của Bảng 2. Kết quả phân tích cho thấy đối với cả hai phần, số nhóm được tách ra là 3[8]. Để kiểm chứng số nhóm có thể tách được, phép phân tích nhóm như trên được lặp lại với toàn bộ mẫu gồm 240 đối tượng. Kết quả có 3 nhóm có thể tách ra. Vậy, số nhóm được tách ra là 3 nhóm và đảm bảo độ tin cậy của sự phân nhóm [11]. Để đánh giá độ giá trị của việc phân nhóm, việc phân nhóm đã được lặp lại dựa trên nguyên tắc tạo sự khác biệt giữa các nhóm với phép đo bình phương khoảng cách Euclide và nguyên tắc tạo sự đồng nhất cao trong mỗi nhóm với phép đo khoảng cách là hệ số tương quan Pearson. Kết quả được trình bày trong 7 cột bên phải của Bảng 2 . Kết quả phân tích cho thấy, đối với cả hai phương pháp phân nhóm với hai phép đo khoảng cách khác nhau, số nhóm được tạo ra đều bằng 3 . Do đó, số nhóm được tạo ra là 3 và giá trị này đảm bảo độ giá trị, độ tin cậy. Để xác định các đối tượng trong mỗi nhóm, phương pháp phân nhóm $\mathrm{K}$ Means đã được thực hiện. Kết quả cho thấy nhóm 1 gồm 72 doanh nghiệp (30\%), nhóm 2 gồm 90 doanh nghiêp (37,5\%), nhóm 3 gồm 78 doanh nghiệp (32,5\%).

Bảng 1: Phân tích nhân tố khám phá và độ tin cậy Alpha

\begin{tabular}{|c|c|c|c|c|c|c|c|c|c|}
\hline & $\begin{array}{c}\text { Hệ số } \\
\text { tải } \\
\text { nhân tố }\end{array}$ & $\begin{array}{c}\text { Phưo'ng } \\
\text { sai trích } \\
(\%)\end{array}$ & $\begin{array}{l}\text { Độ tin } \\
\text { cậy } \\
\text { Alpha }\end{array}$ & $\begin{array}{c}\text { Mã } \\
\text { hóa } \\
\text { thang } \\
\text { đo }\end{array}$ & & $\begin{array}{c}\text { Hệ số } \\
\text { tải } \\
\text { nhân tố }\end{array}$ & $\begin{array}{l}\text { Phưo'ng } \\
\text { sai trích } \\
(\%)\end{array}$ & $\begin{array}{l}\text { Độ tin } \\
\text { cậy } \\
\text { Alpha }\end{array}$ & $\begin{array}{c}\text { Mã } \\
\text { hóa } \\
\text { thang } \\
\text { đo }\end{array}$ \\
\hline \multicolumn{3}{|c|}{ Các đặc điểm của NQLCN } & & & \multicolumn{2}{|c|}{ Sụ tụ chủ } & $66,83 \%$ & 0,750 & V6 \\
\hline \multicolumn{2}{|c|}{ Sụ chấp nhận rủi ro } & $68,40 \%$ & 0,720 & $\mathrm{~V} 1$ & Tuchu22 & 0,763 & & & \\
\hline Ruiro1 & 0,692 & & & & Tuchu23 & 0,850 & & & \\
\hline Ruiro2 & 0,830 & & & & Tuchu24 & 0,837 & & & \\
\hline Ruiro3 & 0,874 & & & & \multicolumn{2}{|c|}{ Quản trị doanh nghiệp } & & & \\
\hline \multicolumn{2}{|c|}{ Nhu cầu thành đạt } & $72,35 \%$ & 0,777 & $\mathrm{~V} 2$ & \multicolumn{2}{|c|}{ Quản trị chiến luợc } & $58,84 \%$ & 0,688 & V7 \\
\hline Thanhdat4 & 0,839 & & & & Cautruc27 & 0,729 & & & \\
\hline Thanhdat5 & 0,849 & & & & Cautruc28 & 0,812 & & & \\
\hline Thanhdat6 & 0,864 & & & & Cautruc36 & 0,758 & & & \\
\hline \multicolumn{2}{|c|}{ Kiểm soát bản thân } & $59,61 \%$ & 0,657 & V3 & \multicolumn{2}{|c|}{ Cấu trúc theo chúc năng } & $54,75 \%$ & 0,644 & V8 \\
\hline Kiemsoat7 & 0,751 & & & & Cautruc33 & 0,717 & & & \\
\hline Kiemsoat8 & 0,822 & & & & Cautruc34 & 0,738 & & & \\
\hline Kiemsoat9 & 0,740 & & & & Cautruc35 & 0,764 & & & \\
\hline \multicolumn{2}{|c|}{ Sụ tụ tin } & $64,08 \%$ & 0,719 & V4 & \multicolumn{2}{|c|}{ Môi trường kinh doanh } & $63,32 \%$ & 0,709 & V9 \\
\hline Tutin15 & 0,819 & & & & Moitruong37 & 0,712 & & & \\
\hline Tutin16 & 0,852 & & & & Moitruong38 & 0,883 & & & \\
\hline Tutin17 & 0,725 & & & & Moitruong39 & 0,783 & & & \\
\hline \multicolumn{2}{|c|}{ Sụ sáng tạo } & $70,23 \%$ & 0,787 & V5 & \multicolumn{2}{|c|}{ Kết quả hoạt động } & $59,66 \%$ & 0,770 & V10 \\
\hline Sangtao18 & 0,777 & & & & Thanhqua40 & 0,763 & & & \\
\hline Sangtao19 & 0,864 & & & & Thanhqua41 & 0,794 & & & \\
\hline \multirow[t]{2}{*}{ Sangtao21 } & 0,870 & & & & Thanhqua42 & 0,710 & & & \\
\hline & & & & & Thanhqua43 & 0,819 & & & \\
\hline
\end{tabular}

\section{Trang 28}


Bảng 2: Kết quả phân tích nhóm

\begin{tabular}{|c|c|c|c|c|c|c|c|c|c|c|c|c|c|}
\hline \multirow{2}{*}{ Bước } & \multicolumn{3}{|c|}{$\begin{array}{c}\text { Một nửa thứ nhất của } \\
\text { mâ̂u }\end{array}$} & \multicolumn{3}{|c|}{$\begin{array}{c}\text { Một nửa thứ hai của } \\
\text { mâ̂u }\end{array}$} & \multirow{2}{*}{ Bước } & \multicolumn{3}{|c|}{$\begin{array}{l}\text { Phương pháp tạo sự } \\
\text { khác biệt nhóm }\end{array}$} & \multicolumn{3}{|c|}{$\begin{array}{l}\text { Phương pháp tạo } \\
\text { đồng nhất nhóm }\end{array}$} \\
\hline & $\begin{array}{c}\text { Nhóm } \\
1\end{array}$ & $\begin{array}{c}\text { Nhóm } \\
2\end{array}$ & Hệ số & $\begin{array}{c}\text { Nhóm } \\
1\end{array}$ & $\begin{array}{c}\text { Nhóm } \\
2\end{array}$ & Hệ số & & $\begin{array}{c}\text { Nhóm } \\
1\end{array}$ & $\begin{array}{c}\text { Nhóm } \\
2\end{array}$ & $\begin{array}{l}\text { Hế } \\
\text { số }\end{array}$ & $\begin{array}{c}\text { Nhóm } \\
1\end{array}$ & $\begin{array}{l}\text { Nhóm } \\
2\end{array}$ & $\begin{array}{l}\text { Hế } \\
\text { số }\end{array}$ \\
\hline 1 & 111 & 112 & 0,436 & 4 & 70 & 0,531 & 1 & 89 & 90 & 0,872 & 148 & 149 & 0,949 \\
\hline
\end{tabular}

\begin{tabular}{|c|c|c|c|c|c|c|c||c|c|c|c|c|c|c|}
\hline 116 & 2 & 75 & 593,907 & 12 & 26 & 696,149 & 237 & 1 & 62 & 27,248 & 1 & 30 & 0,657 \\
\hline 117 & 1 & 4 & 638,346 & 1 & 2 & 743,859 & 238 & 1 & 130 & 33,264 & 1 & 82 & 0,628 \\
\hline 118 & 1 & 2 & 1120,552 & 1 & 3 & 959,485 & 239 & 1 & 148 & 36,317 & 1 & 55 & 0,602 \\
\hline 119 & 1 & 84 & 1294,423 & 1 & 12 & 1062,087 & & & & & & & \\
\hline
\end{tabular}

Kết quả phân tích ANOVA cho sự khác biệt giữa các nhóm về giá trị trung bình của mỗi biến trong mỗi nhóm được trình bày trong Bảng 3 . Kết quả phân tích cho thấy các biến ở các nhóm có sự khác nhau đáng kể ở mức $\mathrm{p}<0.005$ và giá trị của các biến này có thể được xem là những đặc trưng của nhóm. Giá trị trung bình của 10 biến số theo 3 nhóm khác nhau được trình bày dưới dạng đồ thị trong Hình 2. Thống kê đặc điểm nhân khẩu học của NQLCN trong các nhóm được trình bày trong Bảng 4 .

Bảng 3: Kết quả phân tích ANOVA

\begin{tabular}{|c|c|c|c|c|c|c|c|c|c|c|c|c|c|}
\hline \multirow[b]{2}{*}{$\begin{array}{c}\text { Biến } \\
\text { số }\end{array}$} & \multicolumn{2}{|l|}{ Nhóm } & \multicolumn{2}{|l|}{ Sai số } & \multirow[b]{2}{*}{$\mathbf{F}$} & \multirow[b]{2}{*}{ Sig. } & \multirow[b]{2}{*}{ Biến số } & \multicolumn{2}{|l|}{ Nhóm } & \multicolumn{2}{|l|}{ Sai số } & \multirow[t]{2}{*}{$\mathbf{F}$} & \multirow[t]{2}{*}{ Sig. } \\
\hline & $\begin{array}{c}\text { Trung bình } \\
\text { bình } \\
\text { phưo'ng }\end{array}$ & df & $\begin{array}{c}\text { Trung bình } \\
\text { bình } \\
\text { phương }\end{array}$ & df & & & & $\begin{array}{c}\text { Trung bình } \\
\text { bình } \\
\text { phưo'ng }\end{array}$ & df & $\begin{array}{c}\text { Trung bình } \\
\text { bình } \\
\text { phưo'ng }\end{array}$ & df & & \\
\hline V1 & 32,939 & 2 & 0,730 & 237 & 45,093 & 0,000 & V6 & 44,741 & 2 & 0,631 & 237 & 70,918 & 0,000 \\
\hline $\mathrm{V} 2$ & 51,064 & 2 & 0,578 & 237 & 88,420 & 0,000 & V7 & 57,878 & 2 & 0,520 & 237 & 111,298 & 0,000 \\
\hline V3 & 42,536 & 2 & 0,649 & 237 & 65,492 & 0,000 & V8 & 46,840 & 2 & 0,613 & 237 & 76,390 & 0,000 \\
\hline V4 & 55,465 & 2 & 0,540 & 237 & 102,640 & 0,000 & V9 & 53,417 & 2 & 0,558 & 237 & 95,787 & 0,000 \\
\hline V5 & 53,842 & 2 & 0,554 & 237 & 97,173 & 0.000 & V10 & 39,917 & 2 & 0,672 & 237 & 59,438 & 0,000 \\
\hline
\end{tabular}

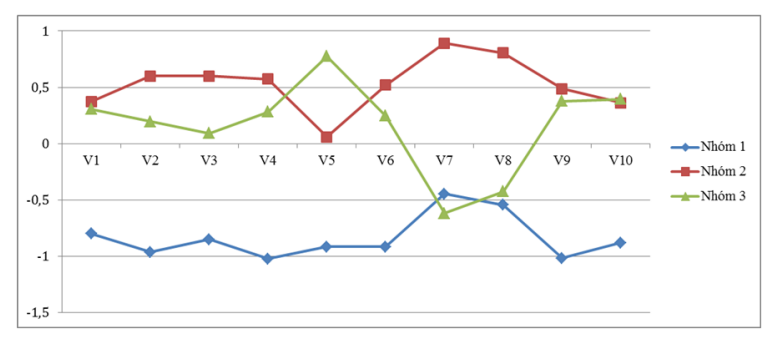

Hình 2: Giá trị trung bình của các biến trong các nhóm 
Bảng 4: Thống kê các đặc điểm nhân khẩu học của NQLCN trong các nhóm

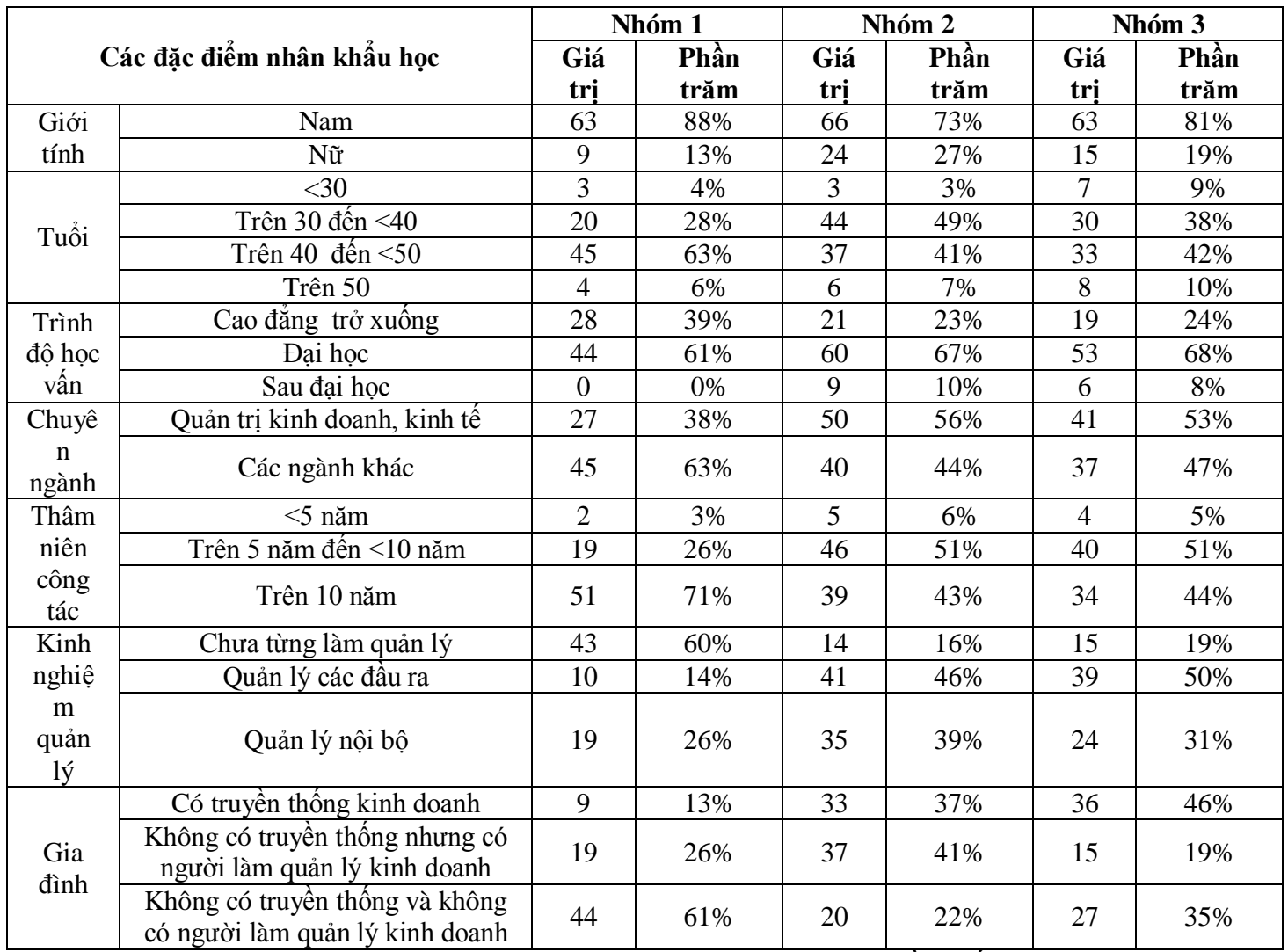

\subsection{Thảo luận}

Sự phân nhóm cho các DNNVV: Nhóm 1 bao gồm NQLCN có TTDN (các biến V1 đến V5) thấp nhất. Nhóm 2 và 3 bao gồm NQLCN có TTDN cao hơn, nhưng nhóm 2 có tính sáng tạo thấp hơn so với nhóm 3 (Hình 2). Nhóm 1 bao gồm các NQLCN lớn tuổi, không được đào tạo trong các chuyên ngành quản lý, kinh tế, chưa từng làm quản lý và gia đình không có truyền thống kinh doanh. Nhóm 2 và 3 phần lớn là NQLCN trẻ, có trình độ học vấn cao hơn nhóm 1 , được đào tạo quản trị kinh doanh, đã từng làm quản lý đầu ra. Sự khác biệt lớn giữa nhóm 2 và 3 là nhóm 2 bao gồm các NQLCN xuất thân từ gia đình không có truyền thống kinh doanh nhưng có người thân làm quản lý, kinh doanh, trong khi nhóm 3 bao gồm các NQLCN xuất thân từ gia đình có truyền thống kinh doanh. Nhóm 1 và nhóm 3 không thực hiện QTCL, xây dựng CT đơn giản, nhóm 2 có thực hiện QTCL và xây dựng CT phân chức năng. Nhóm 1 hoạt động trong môi trường có sự cạnh tranh thấp, tạo kết quả hoạt động thấp. Nhóm 2 và 3 hoạt động trong môi trường có sự cạnh tranh cao, tạo kết quả hoạt động cao (Hình 2). Nhóm 2 có sự sáng tạo gần như bằng 0 và thấp hơn nhóm 3 rất nhiều, nhưng với việc xây dựng $\mathrm{CT}$ theo chức năng và QTCL, nhóm 2 đã tạo kết quả hoạt động tương đương với nhóm 3. Điều này cho thấy $\mathrm{QTCL}$ và xây dựng CT theo chức năng có vai trò quan trọng đối với kết quả hoạt động của doanh nghiệp.

Các dạng thức của NQLCN: Các phân tích nêu trên cho thấy một sự kết hợp của các đặc

\section{Trang 30}


điểm tâm lý, QTCL, CTcủa doanh nghiệp và các đặc điểm nhân khẩu, đặc biệt là truyền thống gia đình, quan hệ xã hội với người làm quản lý kinh doanh sẽ tạo một bộ các tiêu chí để nhận diện dạng thức của NQLCN, phân nhóm các DNNVV. Các nghiên cứu trước đây về dạng thức NQLCN thường chỉ dựa trên đặc điểm tâm lý, nhân khẩu [27], hay phong cách quản lý, QTCL [9], trình độ học vấn, năng lực truyền thông [23] nên tạo các kết quả khác nhau trong việc nhận diện dạng thức của các NQLCN. Bộ tiêu chí được nhận diện trong nghiên cứu này là một bộ tiêu chí tích hợp các đặc điểm tâm lý, nhân khẩu, QTCL và xây dựng $\mathrm{CT}$ theo chức năng của NQLCN, có thể giúp phân nhóm các NQLCN.

Nhận diện quan hệ giữa dạng thức của NQLCN và kết quả hoạt động của DNNVV: NQLCN trong nhóm 1 thường xây dựng doanh nghiệp để sản xuất các sản phẩm họ đã quen thuộc, có kinh nghiệm, chọn sản phẩm trong ngành kinh doanh có sự cạnh tranh thấp và duy trì doanh nghiệp ở mức phát triển trung bình [2]. Do đó, kết quả hoạt động của doanh nghiệp do họ quản lý là thấp và các doanh nghiệp này thuộc dạng "phản ứng" (reactor) [16]. Nhóm 2 bao gồm các NQLCN có tri thức, kinh nghiệm quản lý nên xây dựng $\mathrm{CT}$ theo chức năng. Vì tính sáng tạo thấp, họ theo đuỗi chiến lược nâng cao hiệu quả hoạt động và noi theo các doanh nghiệp khác để sản xuất sản phẩm. Do đó, doanh nghiệp của họ có kết quả hoạt động cao. Các đặc điểm như vậy cho phép phân loại doanh nghiệp thuộc dạng "Phân tích" (analyzer) [16]. Nhóm 3 bao gồm các NQLCN quan tâm đến sáng tạo, cải tiến, thích sự linh hoạt, thay đổi nên tạo các sáng tạo cải tiến đáp ứng với môi trường kinh doanh. Do đó, doanh nghiệp của họ có kết quả hoạt động cao, có nhiều sáng tạo cải tiến. Các đặc điểm nêu trên của doanh nghiệp cho phép phân loại doanh nghiệp thuộc dạng “tìm vàng” (prospector) [16].

\section{KÊT LUẬN VÀ KIẾN NGH!̣}

\subsection{Kết luận}

DNNVV được sáng lập và quản lý bởi NQLCN. Kết quả hoạt động của DNNVV bị ảnh hưởng bởi các đặc điểm tâm lý, nhân khẩu của NQLCN, hệ thống quản lý do họ tạo ra và môi trường kinh doanh. Do tính đa dạng của các yếu tố này, các DNNVV cần thiết được phân nhóm dựa trên sự khác biệt của các yếu tố này. Nghiên cứu này được thực hiện theo định hướng nêu trên nhằm phân nhóm các DNNVV, nhận diện các dạng thức của NQLCN và quan hệ giữa các yếu tố này đến kết quả hoạt động của doanh nghiệp. Kết quả phân tích cho thấy các DNNVV có thể được phân thành 3 nhóm dựa trên sự khác biệt về các đặc điểm tâm lý, nhân khẩu, QTCL và CT theo chức năng. Sự kết hợp các yếu tố này tạo một bộ tiêu chí nhận diện các dạng thức của NQLCN và dự đoán kết quả hoạt động của doanh nghiệp.

\subsection{Kiến nghị}

Hoạt động trong môi trường cạnh tranh cao, NQLCN cần thiết phải nâng cao TTDN, tạo nhiều sáng tạo cải tiến. Đây là hai yếu tố quan trọng để tạo kết quả hoạt động cao cho doanh nghiệp. Việc xây dựng chiến lược, CT theo chức năng sẽ là một hỗ trợ tốt để nâng cao kết quả hoạt động. NQLCN cần thiết phải nâng cao tri thức quản lý, sẽ tạo kết quả hoạt động cao cho doanh nghiệp. Sự xuất thân trong gia đình có truyền thống quản lý, kinh doanh hay có người thân làm quản lý kinh doanh là những thuận lợi cho NQLCN khi thành lập và quản lý doanh nghiệp.

Mặc dù đã nỗ lực nhiều, nghiên cứu này vẫn còn nhiều hạn chế, thiếu sót đặc biệt là việc lấy 
mẫu thuận tiện tại Thành Phố Hồ Chí Minh và chưa khảo sát quan hệ phụ thuộc của kết quả hoạt động của doanh nghiệp đối với các đặc điểm tâm lý nhân khẩu của NQLCN, việc QTCL, CT theo

\section{TÀI LIỆU THAM KHẢO}

[1]. Agarwal, M. N., \& Chatterjee, L., Type of entrepreneur and growth of new ventures: Testing and validation of a typology of software entrepreneurs in India, The Journal of Applied Management and Entrepreneurship, 15, 19 -1 (2014)

[2]. Carland, J. W., Hoy, F., Boulton, W. R., \& Carland, J. A. C., Differentiating entrepreneurs from small business owner: A conceptualization, Academy of Management Review, 9(2), 354-359 (1984)

[3]. Child, J., Organizational structure, environment and performance: The role of strategic choice, Sociology, 6(1), 1 - 22 (1972)

[4]. Child, J., Strategic choice in the analysis of action, structure, organizations and environment: Retrospect and prospect, Organization Studies, 18(43), 43 - 76 (1997)

[5]. Choi, Y. R., \& Shepherd, D. A, Entrepreneurs' decisions to exploit opportunities, Journal of Management, 30(3), 377 - 395 (2003)

[6]. Everitt, B. S., Landau, S., Leese, M., \& Stahl, D, Cluster analysis, 5th Ed.: Wiley (2011)

[7]. Gartner, W. B., A conceptual framework for describing the phenomenon of new venture creation, Academy of Management Review, 10, 696-706 (1985) chức năng, sự lựa chọn môi trường kinh doanh. Các hạn chế này cần thiết được khảo sát trong các nghiên cứu tiếp theo.

[8]. Gartner, W. B., Some suggestions for research on entrepreneurial traits and characteristics, Entrepreneurship Theory and Practice, Fall, 27-37 (1989)

[9]. Gartner, W. B. Variations in entrepreneurship, Small Business Economics, 31, 351 - 361 (2008)

[10]. Hair, J. F. J., Black, W. C., Babin, B. J., \& Anderson, R. E, Multivariate data analysis 7 th Edition: Pearson Prentice Hall. (2010)

[11]. Halkidi, M., Batistakis, Y., \& Vazirgiannis, $\mathrm{M}$, On clustering validation techniques, Journal of Intelligent Information Systems, 17(2/3), 107 - 145 (2001)

[12]. Hambrick, D. C., Upper Echelons Theory: An Update, Academy of Management Review, 32(2), 334 - 343 (2007)

[13]. Tang, J., Tang, Z., Lohrke, F.T., Developing an entrepreneurial typology: The roles of entrepreneurial alertness and attributional style, International Entrepreneurship Management,4,273 - 294 (2008)

[14]. Karadag, H., Financial management challenges in small and medium sizedd enterprises: A strategic management approach, Emerging Market Journal, 5(1), $26-40(2015)$

[15]. Ketchen, D. J., \& Shook, C. L., The application of cluster analysis in strategic

\section{Trang 32}


management research: An analysis and critique, Strategic Management Journal, 17, 441 - 458 (1996)

[16]. Miles, R. E., \& Snow, C. C., Organizational strategy, structure and process: Stanford Business Classics, (2003)

[17]. Miller, D., \& Friesen, P. H., Archetypes of strategy formulation,Management Science, 24(9), 921 - 934 (1978)

[18]. Miller, D., Kets De Vries, M. F. R., \& Toulouse, J. M., Top Executive Locus of Control and its Relationship to Strategy Making, Structure, and Environment, Academy of Management Journal, 25(000002), 237 - 253 (1982)

[19]. Miller, D., \& Toulouse, J. M., Chief executive personality and corporate strategy and structure in small firms, Management Science, 31(11), 1389 - 1410 (1986)

[20]. Miner, J.B. , Testing a psychological typology of entrepreneurship using business founders, The Journal of Behavioral Sciences, 36,43 - 69 (2000)

[21]. Shane, S., \& Venkataraman, S., The promise of entrepreneurship as a field of research, Academy of Management Review, 25(1), 217-226 (2000)

[22]. Sharma, P., Chrisman, J. J., \& Chua, J. H., Strategic management of the family business: Past research and future challenges, Family Business Review, 10(1), 1 - 36 (1997)

[23]. Smith, N. R., \& Miner, J. B., Type of entrepreneur, type of firm and managerial motivation: Implications for organizational life cycle theory, Strategic Management Journal,4, 325 - 340 (1983)

[24]. Verhees, F. J. H. M., \& Meulenberg, M. T. G., Market orientation, innovativeness, product innovation and performance in small firms, Journal of Small Business Management, 42(2), 134-154, (2004) 